\title{
Karl-Dieter Opp Zur Anwendbarkeit der Soziologie im Strafprozeß
}

Seit Jahrzehnten betonen Juristen und auch Soziologen immer wieder, daß die Soziologie zur Lösung juristischer Probleme beitragen kann. Diese These wird durch eine Reihe von Schriften bestätigt, in denen beschrieben wird, wie irgendwelche konkreten juristischen Fragen mittels der Soziologie gelöst oder einer Lösung nähergebracht wurden ${ }^{1}$. Trotzdem kann man ohne Ubertreibung sagen, daß in der Bundesrepublik die Soziologie sowohl bei der Geserzgebung als auch bei der Rechtsprechung ignoriert wird.

Ein Grund hierfür besteht unseres Erachtens darin, daß Juristen normalerweise nicht in der Lage sind anzugeben, bei welcher Art von Problemen welcher »Teil « der Soziologie denn nun anwendbar ist bzw. bei welchen Fragen sie Soziologen zu Rate ziehen könnten. Es fehlt also zunächst ein Problembewußtsein für die Anwendung der Soziologie. Ein zweiter Grund für die Ignoranz gegenüber der Soziologie dürfte darin liegen, daß Juristen nicht wissen oder falsche Vorstellungen darüber haben, welche Antworten genau die Soziologie bieten kann. Wenn Juristen eine Meinung über die Soziologie haben, dürfte die Relevanz der Sozio-

\footnotetext{
1 Der Leser sei insbesondere auf folgende Arbeiten verwiesen, in denen juristisch relevante Fragen mit den Mitteln der Soziologie behandelt werden: A. B. Blumrosen, Antidiscrimination Laws in Action in New Jersey: A Law-Sociology Study, in: Rutgers Law Review 1965, Bd. I9, S. I89-287. Phoebe C. Ellsworth, Robert J. Levy, Legislative Reform of Child Custody Adjudication. An Effort to Rely on Social Science Data in Formulating Legal Policies, in: Law and Society Review 1969, Bd. 4, S. 167-233. J. Feest, Notizen zur Idee einer Kommentierung des $\mathrm{StGB}$ in sozialwissenschafticher Absicht, in diesem Heft S. 457 ff., Harold Goldblatt, Florence Cromien, The Effective Social Reach of the Fair Housing Practices Law of the City of New York, in: Social Problems 196r, Bd. 9, S. 365-70. Burkhart Lutz, Constans Seyfahrt, Informationen zur Situation des Jugendarbeitsschutzes, München 1969. J. Matthes, Gesellschaftspolitische Konzeption im Sozialhilferecht. Zur soziologischen Kritik der neuen deurschen Sozialhilfegesetzgebung 196I, Stuttgart 196r. Helmar Minger, Das Verbor der einfachen Homosexualität im E 62: Eine Kritik aufgrund sozialwissenschafticher Forschungsergebnisse, in: Kriminologisches Journal (erscheint demnächst). Elisabeth Noelle-Neumann, Carl Schramm, Umfrageforschung in der Rechrspraxis, Weinheim 196r. Karl-Dieter Opp, Einige Bedingungen für die Befolgung von Gesetzen, in: Kriminologisches Journal (erscheint demnädist). Adam Podgorecki, Law and Social Engineering, in: Human Organization 1962, Bd. 21, S. 177-8r. Arnold M. Rose, The Social Sciencist as an Expert Witness in Court Cases, in: Paul F. Lazarsfeld, William H. Sewell, Harold L. Wilensky, Hrsg., The Uses of Sociology, London 1967, S. 100-118. Jerome H. Skolnidk, Seientific Theory and Scientific Evidence: An Analysis of Lie Detection, in: Yale Law Journal 1961, Bd. 70, S. 694-729. Hans Zeisel, The Uniqueness of Survey Evidence, in: Cornell Law Quarterly 1960, Bd. 45, S. 322 ff. Ders., The New York Experc Tescimony Project: Some Reflections on Legal Experiments, in: Scanford Law Review 1956, Bd. 8, S. 730-49. Ders., Social Research on the Law: The Ideal and the Practical, in: William M. Evan, Hrsg., Law and Sociology, Glencoe, Ill., 1962, S. 124-44. Ders., The Law, in: Lazarsfeld, Seweil, Wilensky (siehe oben), S. 81-99. Hans Zeisel, Harry Kalven jun., Bernard Buchtolz, Delay in the Court, Boston 1959. Harry Kalven jun., Hans Zeisel, The American Jury, Boston I 966. Leonard Zeicz, Survey of Negroe Acticutes toward Law, in: Rucgers Law Review I965, Bd. I9, S. 288-316.
} 
logie normalerweise unterschätzt werden². Man kann also sagen, daß den Juristen das Problemlösungspotential der Soziologie unbekannt ist.

Wenn man der Meinung ist, daß die Soziologie einen Beitrag zur Lösung praktischer Probleme leisten sollte, erscheint es in der beschriebenen Situation sinnvoll zu zeigen, bei welchen konkreten juristischen Tätigkeiten Soziologie in welcher Weise genau anwendbar ist und welche Antworten der Jurist vom Soziologen erwarten kann. Die meisten juristischen Probleme sind mittels allgemeiner soziologischer "Reflexionen « über Recht und Gesellschaft nicht lösbar ${ }^{3}$. Der Strafrichter will z. B. genau wissen, was ein Soziologe zu der Chance der Rüdkfälligkeit bei einem konkreten Angeklagten zu sagen hat und ob die Ausführungen des Soziologen seiner eigenen "Erfahrung « überlegen sind. Ihm ist wenig damit geholfen, wenn er vom Soziologen hört, daß in allen sozialen Systemen »abweichendes Verhalten vorkommt oder daß die Art und Höhe der Strafe von gesellschaflichen Faktoren beeinflußt wird.

Wir wollen im folgenden versuchen, die Anwendbarkeit der Soziologie im Strafprozeß an einem konkreten Strafrechtsfall zu demonstrieren. Wir werden diesen Fall zunz̈chst darstellen und zeigen, daß die am Strafprozeß beteiligten Juristen implizit fortlaufend allgemeine theoretische Aussagen verwenden. Als nächstes wollen wir prüfen, ob diese von den Juristen verwendeten Theorien soziologischen Theorien widersprechen. $\mathrm{Da}$ dies der Fall ist, fragt es sich, welche der widersprüchlichen Theoriengruppen im Strafprozeß angewender werden soll. Hiermit werden wir uns zum Schluß dieses Aufsatzes befassen. Unser Ziel besteht also erstens darin, auf der Grundlage eines konkreten Beispiels einige allgemeine Hinweise zu geben, wo im Strafprozeß Soziologie anwendbar ist. Weiterhin wollen wir zeigen, daß eine Reihe konkreter juristischer Probleme mittels soziologischer Hypothesen besser als mit den juristischen Alltagstheorien gelöst werden können.

\section{Ein $»$ normaler Strafrechtsfall}

Der im folgenden beschriebene Fall ist im Nürnberger Amtsgericht Ende Mai 1970 verhandelt worden. Angeklagt war eine Frau, etwa 45 Jahre, wegen Diebstahls in Mittäterschaft. Thr wurde also zur Last gelegt, zusammen mit einem anderen Täter eine fremde bewegliche Sache weggenommen zu haben in der Absichs, sich diese Sache rechtswidrig zuzueignen $(\$ \$ 242,47 \mathrm{StGB})$.

Die Angeklagte beschreibt den Tathergang aufgrund von Fragen des Ridters in folgender Weise. Sie habe mit einem Mann, den sie einige Tage vorher kennengelernt habe, an einem Tisch in einer Gastwirtschaft in einem verrufenen Viertel in Nürnberg gesessen. An diesen Tisch sei nach einiger Zeit Herr B - der Bestohlene - gekommen. Dieser sei bereits stark angetrunken gewesen und nach einiger Zeit am Tisch eingeschlafen. Dann habe die Angeklagte gesehen, daß die Geidbörse des Herrn B etwas aus der hinteren Hosentasche herausragte. Auch der Begleiter der Angeklagten habe die Geldbörse erblickt und gesagt, sie solle diese doch einmal herausnehmen. Dies sei geschehen. Ihr Begleiter babe ihr dann die Geldbörse aus der Hand genommen oder gerissen, sie habe ihm die Geldbörse jedenfalls nicht gegeben. Dann sei ihr Begleiter ohne sie aus der Wirt-

2 Vgl. hierzu etwa die Untersuchung von: Richard H. Blum, Mary Lou Funkhouser, Legislators on Social Scientists and a Social Issue, in: Journal of Applied Behavioral Sciences 1965, Bd. $\mathrm{x}, \mathrm{S} .84-\mathrm{r} \mathbf{2}$.

3 Vgl. hierzu auch die Kritik von: Wolfgang Naudse, Ober die Zusammenarbeit zwischen Juristen und Rechtssoziologen, in: Rüdiger Lautmann, Werner Maihofer, Helmut Schelsky, Hrsg. Die Funktion des Rechrs in der modernen Gesellschaft, Jahrbuch für Rechrssoziologie, Bd. I, Bielefeld 1970 , S. $492-97$. 
schaft gegangen. Sie bestreitet, seit diesem Zeitpunkt ihren Begleiter noch einmal gesehen zu haben; sie sei auch nach der Entnahme der Geldbörse nicht nach draußen gegangen und habe sich dort mit ihrem Begleiter getroffen. Von dem Geld, das sich in der Geldbörse befand - nach Auskunft des Bestohlenen $600 \mathrm{DM}$ - habe sie nichts bekommen. Sie habe auch dem Bestohlenen das Geld nicht stehlen wollen. Sie wisse zwar nicht, warum sie die Geldbörse aus der Tasche gezogen habe, jedenfalls nicht, um sich das Geld zuzueignen. Weiterhin hätte sie zwei schmerzstillende Tabletten genommen und einige Gläser Alkohol getrunken, bevor sie die Geldbörse aus der Tasche des Bestohlenen genommen habe. Sie gibe dem Gericht weiterhin an, wie ihr Begleiter heißt. Seinen Namen hatre sie niche gekannt und inzwischen ausfindig gemacht. Die Angeklagte war zomal porbestraft, davon einige Male einschlägig. In den Jahren I958-68 ist kein Verfahren gegen sie eröf net worden. Während dieser Zeit war sie verheiratet. Mitrlerweile ist sie geschieden. Sie hat to Kinder; 8 Kinder sind in einem Heim, 2 verheirater. Gegenwärtig ist sie Prostituierte. Vorher war sie eine Zeitlang Keilnerin. Sie ist haftunfähig.

Weiterhin wurde eine Zeugin vernommen, die die Anzeige erstattete. Sie behauptete, die Angeklagte habe die Gaststätte verlassen, kurz nachdem ihr Begleiter ihr die Geldbörse aus der Hand genommen habe.

Ein Zeuge sagt, er könne sich nicht daran erinnern, ob die Angeklagte aus dem Lokal gegangen sei oder nicht, nachdem der Begleirer ihr die Geldbörse weggenommen hat. Er sagt, er halte dies aber nicht für möglich, da die Angeklagte zwischen Stühlen eingeklemmt war und sich somit kaum hätte entfernen können, ohne daß er das bemerkt hätte.

Der Bestohlene kann sich an den Tathergang nicht erinnern, da er am Tisch fest eingeschlafen war. Alle Beteiligten hatten mehrere Gläser Bier und einige Schnäpse zu sich genommen.

Der Richter erwecste den Eindruds, daß er von vornherein der Meinung war, die Angeklagte habe sich das Geld zueignen wollen. Er stellte sehr häufig Suggestivfragen, d. h. die Formulierung einer Frage ließ die Antwort, die der Richtcr erwartete, erkennen. So *fragtex er: „Geben Sie doch zu: Wollten Sie das Geld stehlen und haben Sie den Raum verlassen, um mit ihrem Begleiter zu teilen? \& Einen groben Eindrudk von dern Verhalten des Richters mag der Leser bekommen, wenn man ihn als unfreundlich und teilweise unverschämt bezeidhnet. So unterbrach er die Angeklagte mehrfach wie ein autoritärer Lehrer einen Schüler, den er am liebsten von der Schule verweisen möchte. Zu der rojährigen Straflosigkeit der Angeklagten sagte er z. B., daß er sich dies eigentlich nidht vorstellen könne; er babe zuerst geglaubt, im Strafregister habe eine Seite gefehit.

Der Staatsanwalt beantragte eine Gefängnisstrafe von s Monaten ohne Bewährung und Auferlegung der Gerichtskosten. Der Richter verurteilte die Angeklagte zu 4 Monaten Gefängnis ohne Bewährung und zur Zahlung der Gerichtskosten. Er sah also den Tatbestand des Diebstahls als erfüllt an. Im Urteil hieß es u. a., die Angeklagte habe durch ihre rojährige Straflosigkeit bewiesen, daß sie sich gesetzmäßig verhalten könne, so daß sie sich eine empfindliche Strafe wahrscheinlich zur Warnung dienen ließe.

Es mag von Interesse sein, daß diese Verhandlung von etwa is Teilnehmern eines Seminars über Rechtssoziologie, das im Sommersemester 1970 in Nürnberg stattfand, besucht wurde. Alle Teilnehmer waren über das Verhalten des Richters entrüstet. Niemand hätte die Angeklagte verurteilt.

\section{Alltagstheorien des Richters: Versuch einer Rekonstruktion}

Wir sahen, daß der Richter den Tatbestand des Diebstahls als erfüllt ansah, anders gesagt: Der Richter hielt den Satz $\gg$ Frau $S$ hat zum Zeitpunkt $t$ am Ort O einen Diebstahl begangen « für wahr. Es handelt sich hier um einen singulären Satz - bezeichnen wir ihn als So (S-null) -, d. h. um einen Satz, der für einen 
bestimmten Ort und für einen bestimmten Zeitpunkt oder Zeitraum gilt. Aus welchen Gründen hat nun der Richter diesen Satz akzeptiert und nicht die Negation dieses Satzes, d. h. daß die Angeklagte den Diebstahl nicht begangen hat? Hinweise zur Beantwortung dieser Frage gibt das Verhalten des Richters während der Verhandlung. Er stellte der Angeklagten und den Zeugen eine Reihe von Fragen, mit denen er feststellen wollte, ob bestimmte Fakten vorlagen. Anders gesagt: Er versuchte zu ermitteln, ob bestimmte andere singuläre Sätze wahr sind. So versuchte der Richter herauszufinden, ob die Angeklagte den Raum verlassen und wieviel Alkohol sie zu sich genommen hatte. Weiterhin ließ er sich genau beschreiben, wie der Begleiter der Angeklagren die Geldbörse erhielt; ob ihm die Angeklagte die Geldbörse gab oder ob er der Angeklagten die Geldbörse abnahm. Der Richter ging davon aus, daß die Angeklagte die Geldbörse dem Bestohlenen aus der Tasche genommen hatte und er versuchte zu ermitteln, "warum" die Angeklagte die Geldbörse dem Bestohlenen aus der Tasche zog. Der Richter fragte auch nach dem Einkommen der Angeklagten. Offenbar hielt der Richter u. a. folgende singuläre Sätze für wahr:

S I: Die Angeklagte hat die Geldbörse dem Bestohlenen aus der Tasche gezogen; $S_{2}$ : Die Angeklagte hat ein Einkommen von $x$ DM monatlich;

$\mathrm{S}_{3}$ : Die Angeklagte gibt keinen Grund für die Wegnahme der Geldbörse an;

$\mathrm{S}_{4}$ : Vor der Wegnahme der Geldbörse hat die Angeklagte $x$ Gläser Bier und y Gläser Schnaps und zwei schmerzstillende Tabletten zu sich genommen.

Schließlich entnahm der Richter vorliegenden Urkunden, daß die Angeklagte vorbestratt ist, so daß er folgenden Satz für wahr hält:

$S_{S}:$ Die Angeklagte ist $30 \mathrm{mal}$ vorbestraft, davon einige Male einschlägig.

Warum ist nun der Richter daran interessiert zu wissen, ob diese Sätze wahr sind? Offenbar glaubt er, daß die Fakten, die durch die Sätze $S_{\text {I }}$ bis $S$ s bezeichnet werden, darauf »schließen « lassen, daß der zuerst genannte singuläre Satz wabr ist, daß also die Angeklagte die Absicht hatte, sich das Geld zuzueignen. Anders gesagt: er nimmt offenbar kausale Beziehungen $z$ wischen den durch $S_{I}$ bis $S_{S}$ bezeichneten Fakten und dem durch So bezeichneten Tatbestand an. Der Richter würde erwa sagen: »Weil « die Angeklagte dem B die Geldbörse aus der Tasche zog, ist mit einer gewissen Wahrscheinlichkeit anzunehmen, daß sie sich diese Geldbörse zueignen wollte. »Weil « sie einschlägig vorbestraft ist, »weil « sie auf die Frage, warum sie die Geldbörse aus der Tasche genommen hat, keinen Grund angibt und "weil « schließlich ihr Einkommen gering ist, kann angenommen werden, daß sich die Angeklagte die Geldbörse zueignen wollte. Dies wird auch durch den Alkoholkonsum nicht ausgeschlossen, da er ziemlich gering war. Man könnte die Beziehungen zwischen den Fakten, die nach der Meinung des Richters vorlagen, in folgendem Graphen darstellen:

Warum sind nun nach der Meinung des Richters die durch die Sätze bezeichneten Fakten für das Vorliegen der Absicht, sich die Geldbörse zuzueignen, von Bedeutung? Warum hat der Richter sich nicht darüber informiert, ob die Angeklagte den Blinddarm noch hat oder ob sie zum Frühstüdk Marmelade oder Wurst gegessen hat? Der Richter würde antworten, daß die beiden genannten Fakten völlig irrelevant sind für die Frage, ob sich die Angeklagte die Geldbörse zueignen wollte oder nicht. Warum sind diese beiden und andere Fakten irrelevant? Der Richter würde wahrscheinlich sagen, daß man dies aus Erfahrung wisse: Wenn jemand einem anderen eine Geldbörse aus der Tasche zieht, ein geringes 
Einkommen hat, keinen Grund für die Entnahme angeben kann und dazu noch vorbestraft ist, dann ist anzunehmen, daß diese Person sich die Geldbörse zueignen wollte.

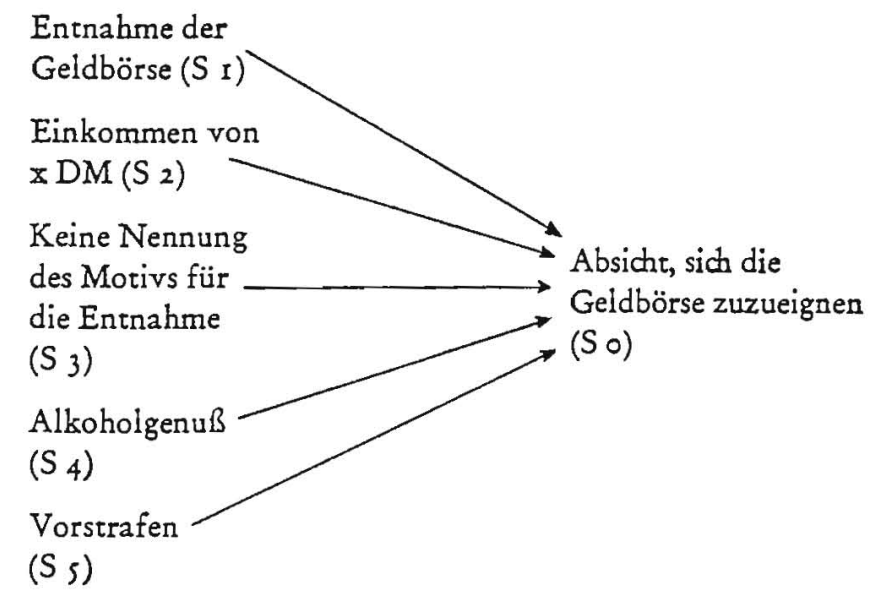

Rekonstruieren wir die Vorgehensweise des Richters nun etwas genauer. Er hat aus der Fülle der vorliegenden Fakten einige wenige Fakten herausgegriffen und in eine Beziehung zueinander gesetzt. Welche Fakten für welche anderen Fakten relevant sind, d.h. aufgrund welcher Fakten man auf das Vorliegen anderer Fakten »schließen k kann, weiß der Richter aus Erfahrung, genauer gesagt: Er wendet allgemeine, d. h. nicht singuläre empirische Sätze (Erfahrungssätze) an, die er für wahr hält. Derartige Sätze bezeichnet man ais wahre Theorien oder Hypothesen.

In ihrer einfachsten Form sind solche Theorien Aussagen der Art »wenn eine bestimmte Klasse von Tatbeständen vorliegt, dann liegt (mit einer mehr oder weniger großen Wahrscheinlichkeit) eine bestimmte andere Klasse von Tatbeständen vor«. Man bezeichnet diese Sätze auch als wenn-dann-Sätze. Sie bestehen also aus zwei Komponenten: der wenn-Komponente, in der Bedingungen für das Auftreten anderer Tatbestände genannt sind, und der dann-Komponente, in der bestimmte Wirkungen $\ll$ der Bedingungen behauptet werden. Diese Aussagen sind empirisch, sie können also falsch oder auch wahr sein.

In welcher Beziehung stehen nun die Fakten, die der Richter ermittelt hat, und die Theorien, die der Richter angewendet hat? Die konkreten Fakten, die nach der Meinung des Richters die Bedingungen für das Vorliegen anderer Fakren sind, gehören zu den durch die wenn-Komponente bezeichneten Tatbeständen. So hat unser Richter die Vorstrafen der Angeklagten ermitteit, weil allgemein Vorstrafen „darauf schließen lassen «, daß ein Diebstahl stattgefunden hat. Die Fakten, die also als relevante Bedingungen für das Vorliegen anderer Fakten betrachtet werden, sind unter die wenn-Komponente der angewendeten Theorien subsumierbar. Man nennt solche Fakten Randbedingungen oder auch $A n$ fangsbedingungen. Die Fakten dagegen, auf die »geschlossen« wird, sind "Teile der dann-Komponente. Man nennt sie deshalb Explananda, d. h. Tatbestände, deren Auftreten zu erklären ist. Die Beziehungen zwischen den Fakten, die durch die singulären Sätze bezeichnet werden, und den angewendeten Theorien lassen sich schematisch in folgender Weise darstellen:

4 Zur Struktur solcher Theorien vgl. genauer meine Schrift: Merhodologie der Sozialwissenschaften. Einführung in Probleme ihrer Theorienbildung, Reinbek r970, S. 45-50. 


\begin{tabular}{|c|c|}
\hline $\begin{array}{l}\text { Wenn: } \\
\text { Entnahme, Einkommen x, } \\
\text { keine Nennung des Motivs, } \\
\text { geringer Alkoholgenuß, } \\
\text { Vorstrafen, } \\
\text { Randbedingungen: } \\
\text { bei der Angeklagten lagen vor: } \\
\text { Entnahme, Einkommen x, keine } \\
\text { Nennung des Motivs ... }\end{array}$ & $\begin{array}{l}\text { dann (immer oder meistens): } \\
\text { Absicht ... }\end{array}$ \\
\hline & $\begin{array}{l}\text { Explanandum: } \\
\text { Die Angeklagte hatte die Abs }\end{array}$ \\
\hline
\end{tabular}

Aus diesem Schema ${ }^{5}$ geht hervor, daß der Richter aus der Theorie und den Sätzen, die die Randbedingungen bezeichnen, logisch schließen kann, daß das Explanandum vorliegt, genauer, daß der Satz, der das Explanandum bezeichnet, wahr ist.

Aus unseren Ưberlegungen ergibt sich also folgendes. Das Ziel des Richters bestand darin festzustellen, ob ein bestimmter singulärer Satz So wahr ist, ob nämlich die Angeklagte die Absicht hatte, sich die Geldbörse zuzueignen oder nicht. Dieses Faktum hat der Richter nicht direkt ermittelt, sondern er hat andere Fakten erhoben, von denen aus er auf das Vorliegen des durch So bezeichneten Faktums schloß, genauer: er hat eine Theorie angewendet, unter deren dann-Komponente die durch So bezeichneten Fakten subsumierbar sind. Er hat dann die Fakten erhoben, die unter die wenn-Komponente subsumierbar sind und logisch geschlossen, daß der zu ermittelnde Sachverhalt vorlag. Die angewendete Theorie gab dem Richter also gewissermaßen Hinweise darauf, welche Fakten für das Auftreten des Explanandums relevant sind.

Die in unserem Beispiel angewendete Theorie war eine wenn-dann-Aussage. Diese ist - wie wir sagten - die einfachste Form einer Theorie. Man bezeichnet auch empirische Särze der Art "je größer X, desto größer $Y_{*}$ als Theorien. So wäre z. B. die Aussage »je härter ein Angeklagter bestraft wird, desto geringer ist die Rückfallwahrscheinlichkeit* ebenfalls eine Theorie. Unsere vorangegangenen Ausführungen gelten auch für solche je-desto-Sätze, so daß wir uns hier nur mit der einfacheren Form von Theorien zu befassen brauchen.

Wir haben bisher nur einen kleinen Ausschnitt des Verhaltens des Richters beschrieben und gesehen, daß er in bestimmter Weise eine Theorie angewendet hat. Analysiert man die Vorgehensweise des Richters weiter, dann zeigt sich, daß er offenbar - bewußt oder unbewußr - eine Vielzahl von Theorien anwendet. Einige Beispiele mögen dies illustrieren. Wir sagten, der Richter habe Suggestivfragen gestellt. Warum? Vielleicht nahm er an, daß er hierdurch eher die Wahrheit erfährt. Er könnte die Theorie angewendet haben: »Wenn man einem Angeklagten Suggestivfragen stellt, dann ist die Wahrscheinlichkeit hoch, daß er die Wahrheit sagt.« Der Richter hat nicht daran gezweifelt, daß das ihm vorliegende Strafregister wahre Angaben enthält. Wenn die Angeklagte diese Angaben bestritten hätte, würde ihr der Richter sicherlich nicht geglaubt haben. Wahrscheinlich hat er die Theorie angewendet: » Wenn eine Behörde eine Urkunde ausfertigt, dann ist diese Urkunde fehlerlos.« Der Richter hat sich mit der

\footnotetext{
' Es handeit sich hier um das sog. Hempel-Oppenheimsche Erkiärungsschema. Zusammenfas-
} send vgl. meine in Fußnote 4 genannte Schrift. 
Angeklagten so unterhalten, als ob sie die Bedeutung seiner Außerungen versteht. Auch hier hat er wiederum sein $\gg$ Erfahrungswissen $*$ angewender, d. h. eine Theorie, die man erwa folgendermaßen skizzieren könnte: "Wenn eine Person deutsche Staatsangehörige ist, wenn sie in bestimmter Weise auf Fragen antwortet, dann versteht sie die deutsche Sprache $\alpha$. Auch bei der Beurteilung der Glaubwürdigkeit irgendwelcher Personen werden Theorien angewendet. Der Richter. hat der Angeklagten geglaubr, daß sie gegenwärtig als Prostituierte arbeitet. Warum? Vielleicht hat der Richter folgende Theorie angewendet: "Wenn jemand eine Beschäftigung angibt, die ein sehr starkes negatives Prestige hat, dann hat er diese Beschäftigung.^ Der Richter sagte in der Urteilsbegründung, daß seiner Meinung nach die Strafe die Angeklagte von weiteren Straftaten abhalten könnte, da sie lange Zeit straflos gewesen sei. Es scheint, daß er eine Variante der bereits genannten Theorie anwandte: Wenn ein Angeklagter mehrere Jahre straflos war, gilt: je höher der Angeklagte bestraft wird, desto geringer ist die Rückfallwahrscheinlichkeit. « In dieser Hypothese müßte der Ausdruck *mehrere Jahre präzisiert werden.

Wir haben uns darauf beschränkt, die Vorgehensweise des Richters zu analysieren, da der Staatsanwalt kaum in die Verhandlung eingegriffen hat. Aus der Begründung der Anklage und dem Strafantrag ergibt sich, daß auch der Staatsanwalt eine Vielzahl von Theorien angewendet hat.

Versuchen wir nun, in allgemeiner Weise zu beschreiben, wie der Richter Theorien angewendet hat. Zunächst will der Richter wissen, ob ein bestimmter Tatbestand (das Explanandum) vorlag, vorliegt oder vorliegen wird; ob z. B. die Angeklagte die Absicht hatte, sich die Geldbörse zuzueignen, ob ein Zeuge glaubwürdig ist oder ob die Angeklagte rückfüllig werden wird. Sodann sucht er eine empirische Theorie, zu deren dann-Komponente diese Tatbestände gehören. Die wenn-Komponente dieser Theorie gibe dem Richter dann die Information, unter welchen Bedingungen das Explanandum allgemein vorliegt. Drittens ermittelt der Richter dann im konkreten Fall, ob die Bedingungen vorliegen (ob etwa die Angeklagte vorbestraft ist oder nicht), oder er produziert die Bedingungen (er stellt z. B. suggestive Fragen); d. h. der Richter erhebt oder produziert die Randbedingungen. Liegen diese vor, schließt der Richter aus der Theorie und den Randbedingungen auf das Explanandum ${ }^{6}$.

Genau in dieser Weise gehen auch der Staatsanwalt und die anderen am Strafprozeß beteiligten Personen vor. Selbstverständlich dürfte die Vorgehensweise kaum einem Richter, Staatsanwalt usw. bewußt sein.

Der Leser mag bezweifeln, daß der Richter tatsächlich in dem Ausmaß empirische Theorien angewendet hat, wie wir es hier behauptet haben. Man hätte dies prüfen können, indem man den Richter gefragt hätte, warum er bestimmte Tatbestände erhoben hat und andere nicht oder warum er sich in bestimmter Weise und nicht anders verhalten hat. Man hätte ihn weiterhin bei einer Reihe verschiedener Theorien, die aufgrund seiner Äußerungen rekonstruiert wurden, fragen können, ob er diese Theorien für richtig hält. Wir versuchten, den Richter zu einer Diskussion im Rahmen des bereits genannten Seminars über Rechtssoziologie einzuladen. Leider lehnte er ab mit der Begründung, daß er sich mit dem neuen Strafrecht noch nicht so gut auskenne und nicht in der Lage sei, entsprechende Fragen zu beantworten. Er sagte auch dann nicht zu, als wir ihm ver-

- Diese Vorgehensweise wird zuweilen mehrfach hintereinander durchgeführe. D. h. die Randbedingungen werden wiederum als Explanandum verwender u. S. w. In inserem Beispiel war die Randbedingung - Vorstrafen der Angeklagten wiederum Explanandum der Theorie $\rightarrow$ Wenn eine Behörde cine Urkunde ausfertige, dann ist diese Urkunde fehierlos*. 
sicherten, daß solche Fragen nicht gestellt werden, sondern daß wir vor allem sein Verhalten während des Prozesses diskutieren wollten, u. a. also auch, welche empirischen Theorien er implizit bei dem beschriebenen Prozeß anwandte.

Ohne die Befragung des Richters ist es sicher schwierig, genau die Art der von ihm angewendeten Theorien zu rekonstruieren. Es erscheint jedoch wenig zweifelhaft, daß der Richter eine Vielzahl von empirischen Theorien anwandte. Wir sehen keine alternative Möglichkeit zu erklären, warum der Richter ganz bestimmte konkrete Fakten für relevant, andere für irrelevant hält. Wenn er z. B. nicht daran interessiert ist, die Schuhgröße der Angeklagten, sondern ihren Alkoholgenuß vor der Tat zu ermitteln, dann fragt es sich, warum er gerade u. a. aufgrund dieser Tatbestände schließen zu können glaubt, daß die Angeklagte die Absicht hatte, sich die Geldbörse zuzueignen.

Fassen wir das Ergebnis unserer Ausführungen zusammen. Wir sahen, daß der Richter sowohl bei der Ermittlung des Sachverhalts als auch bei der Begründung des Urteils eine Vielzahl von empirischen Theorien in bestimmter Weise anwendet. Diese Theorien wurden nicht explizit formuliert, sie wurden vielmehr nur angedeutet.

Unsere Ausführungen bezogen sich auf einen einzigen Strafprozeß. Das Ergebnis dieser Analyse dürfte jedoch für alle Strafprozesse zutreffen. Auch hier haben Richter, Staatsanwalt und evtl. Verteidiger, Schöffen und Geschworene bestimmte Vorstellungen darüber, welche Tarbestände für welche anderen Tarbestände Bedingungen sind und wie bestimmte Maßnahmen - sei es ein Urteil, eine Vereidigung, eine konkrete Frage z.B. an einen Zeugen oder eine Behauptung wirken, d. h. es wird mehr oder weniger implizit eine Vielzahl von Theorien angewendet. Es ist also ein Irrum zu glauben, daß nur dort empirische Theorien ins Spiel kommen, wo ein Gutachter hinzugezogen wird oder wo explizit auf die »Erfahrung « verwiesen wird.?

\section{Eine Alternative: Die Anwendung sozialwissenschaflicher Theorien}

Die im Strafprozeß angewendeten Theorien spielen für die verschiedenen Entscheidungen der Prozeßbeteiligten eine wichtige Rolle. Dies gilt vor allem für zwei Entscheidungen des Richters: Die Entscheidung, ob der geserzliche Tatbestand erfülit ist oder nicht und ggfs. die Strafzumessung. Dies läßr sich an unserem Beispiel zeigen: Aufgrund der angewendeten Theorien kam der Richter zu dem Ergebnis, daß die Angeklagte die Absicht hatte, sich das Geld zuzueignen. Dieses Ergebnis war in unserem Falle eine notwendige Bedingung dafür, daß der Richter den gesetzlichen Tatbestand des Diebstahls als erfüllt ansah. Damit wird die Angeklagte den im Gesezz fixierten Rechtsfolgen unterworfen. Die Theorien des Richters spielten zweitens eine Rolle für die Strafzumessung: $E_{r}$ glaubte, daß die von ihm verhängte Strafe eine »Besserung « der Angeklagten bewirken würde.

Wenn nun die angewendeten Theorien falsch sind, wird man auch die Entscheidungen, die vor allem aufgrund dieser Theorien getroffen wurden, als Fehlentscheidungen bezeichnen, $d$. $h$. man würde - falls man die wahren Theorien wüßte - andere Entscheidungen treffen. Wenn etwa in unserem Beispiel die genannten

\footnotetext{
7 Andere Beispiele für im Strafprozeß angewendece Theorien über Rüokfülligkeic enchäic die folgende Schrift Rüdiger Peudert und Karl-Dieter Opp, Ideologie und Fakren in der Rechtsprechung. Eine soziologische Untersuchung über das Urteil im Strafprozeß, München $197 x$.
} 
Bedingungen, die nach der Meinung des Richters dafür sprechen, daß die Angeklagte die Absicht hatte, sich das Geld zuzueignen, in Wirklichkeit überhaupt keinen Schluß auf die Absicht der Angeklagten zulassen; wenn dagegen die wahren Theorien bekannt wären und wenn weiterhin aus diesen und vorliegenden Randbedingungen hervorgeht, daß die Angeklagte nicht die Absicht hatte, sich die Geldbörse zuzueignen, wird man die Behauptung des Richters, die Angeklagte habe die genannte Absicht gehabt, nicht akzeptieren. Man wird das Urteil des Richters als ein Fehlurteil bezeichnen ${ }^{8}$. Wenn weiterhin die Höhe der vom Richter festgesetzten Strafe genau die entgegengesetzte Konsequenz hat als die, die der Richter annimmt, wird man auch das Strafmaß nicht akzeptieren.

Wir sehen also, daß man eine Vielzahl richterlicher Entscheidungen nur dann als akzeptabel bezeichnen wird, wenn die angewendeten Theorien wahr sind. Es scheint nun, daß die am Strafprozeß beteiligten Juristen glauben, daß ihre Theorien wahr sind; denn da falsche Theorien zu nicht akzeptablen Entscheidungen führen, ist anzunehmen, daß man nur solche Theorien anwender, die man für wahr hält.

Was veranlaßt nun die am Strafprozeß beteiligten Juristen dazu zu glauben daß die angewendeten Theorien wahr sind? Wie kommt z. B. der Richter in dem beschriebenen Prozeß dazu anzunehmen, daß ein Vorbestrafter eher als ein Nicht-Vorbestrafter eine Straftat, deretwegen er angeklagt ist, begangen hat? Woher glaube der Richter zu wissen, daß die Höhe der Strafe negativ korreliert mit der Rückfallwahrscheinlichkeit? Wenn man behaupten würde, daß suggestive Fragen gerade nicht dazu geeignet sind, die Wahrheit herauszufinden: Wie würde der Richter hierauf antworten, d.h. wie würde er argumentieren, um die genannte Behauptung, die er offenbar nicht akzeptiert, zu widerlegen?

Wie ein am Strafprozeß beteiligter Jurist diese Fragen auch immer beantworten mag - wir kommen darauf noch zurück -: Er ignoriert vorliegende Theorien, die von Soziologen formuliert und durch empirische Untersuchungen geprüft und bestätigt wurden. Daß diese Theorien ignoriert werden, dürfte kaum bestritten werden: Sie werden bei der Ausbildung des Juristen nicht vermittelt; auch in den Urreilen wird normalerweise keine soziologische Literatur ziriert.

Die Nicht-Anwendung sozialwissenschafticher Theorien im Strafprozeß könnte nun dadurch bedingt sein, daß keine Theorien existieren, die aufgrund ihrer logischen Struktur im Strafprozeß anwendbar sind und vom Richter (oder anderen am Strafprozeß beteiligten Juristen) benötigt werden. D.h. es wäre möglich, daß Theorien mit den für den Richter relevanten Randbedingungen und Explananda in der Soziologie nicht vorliegen. Um zu entscheiden, ob dies zutrifft oder nicht, müßte man zunächst ein Inventar der im Strafprozeß relevanten Randbedingungen und Explananda aufstellen. Dies ist selbstverständlich in diesem Rahmen nicht möglich. Wir wollen uns vielmehr darauf beschränken zu zeigen, daß für eine Reihe von Randbedingungen und Explananda, die im Strafprozeß relevant sind, soziologische Hypothesen existieren.

Richter und Staatsanwalt befassen sich in einem Strafprozeß zu einem großen Teil damit, Angeklagte, Zeugen und evt. auch Gutachter zu befragen. Eine in der Soziologie sehr häufig verwendete Forschungstectnik ist das Interview ${ }^{\natural}$. $\mathrm{Zu}$

\footnotetext{
8 Es wäre einmal interessant zu untersuchen, inwieweit die bekanntgewordenen Fehlurteile durch falsche Theorien bedingt wurden und wie diese Theorien genau lauten. Material für eine solche Analyse enthalten die beiden folgenden Bücher: Max Hirschberg, Das Fehlurteil im Strafprozeß, Stuttgart 1960. Karl Peters, Fehlerquellen im Strafprozeß, 1. Band, Karlsruhe 1970. - Vgl. hierzu folgende deutschsprachige Beiträge: Erwin K. Scheuch, Das Interview in der So-
} 
dieser Forschungsmethode gibr es eine Vielzahl von Hypothesen darüber, wie sich das Verhalten eines Interviewers, der Aufbau des Fragebogens, die Formulierung der einzelnen Fragen usw. auf das Verhalten des Befragten auswirken und auch darüber, wie das Verhalten des Befragten das Verhalten des Interviewers beeinflußr. So finden wir z. B. Untersuchungen über die Wirkung suggestiver Fragen, über die Wirkung verschiedener Fragearten und über die Wirkung bestimmter sozialer Merkmale des Interviewers - wie Schichtzugehörigkeit, Geschlecht usw. - auf das Verhalten des Befragten. Es wäre möglich, diese Hypothesen auch im Strafprozeß anzuwenden, selbst wenn die "normale « Interviewsituation und der Strafprozeß sich in einer Reihe von Merkmalen unterscheiden. Der Grund ist, daß es Hypothesen gibt, in denen die Wirkung des Interviewerverhaltens unter verschiedenen Bedingungen erklärt und vorausgesagt werden kann - etwa wenn wincime « Fragen beantwortet werden sollen -, die im Strafprozeß realisiert sind oder realisiert werden könnten.

Wir sagten, daß der Richter bei einem Urteil u. a. die Wirkung seines Urteils und die "Ursachen« für die Tat des Angeklagten berüdksichtigt. Hierzu gibt es eine Reihe von Hypothesen im Rahmen der Soziologie des abweichenden Verhaltens ${ }^{10}$.

Diese Beispiele mögen genügen, um zu zeigen, daß die Ignoranz soziologischer Theorien durch die am Strafprozeß beteiligten Juristen nicht dadurch bedingt sein kann, daß es hier keine Theorien gibr, die im Strafprozeß anwendbar sind. Es wäre jedoch denkbar, daß es zwar im Strafprozeß anwendbare Theorien der Soziologie gibt, daß sich diese jedoch von den Theorien, die Juristen im Strafprozeß verwenden, nicht unterscheiden. Ob dies der Fall ist oder nicht, wurde $z$ war von Juristen nicht geprüft. Trotzdem könnte eine Ubereinstimmung $z$ wischen den verwendeten Theoriengruppen bestehen.

Wie hoch der Grad der Ubereinstimmung genau ist, läßt sich nur nach eingehenden Analysen entscheiden. Wenn wir annehmen, daß die von *unserem « Richter angewendeten Theorien auch von anderen Richtern angewendet werden ${ }^{11}$, läßt sich aufgrund des beschriebenen Prozesses zeigen, daß soziologische Theorien zumindest nicht immer mit den im Strafprozeß angewendeten Theorien übereinstimmen. Demonstrieren wir dies an einigen Beispielen.

Der Richter fragte die Angeklagte, warum sie die Geldbörse dem Bestohlenen aus der Tasche genommen habe. Die Angeklagte konnte diese Frage nicht beantworten. Wir sagten, daß dies aufgrund der vom Richter angewendeten Theorie die Wahrscheinlichkeit erhöhte, daß die Angeklagte die Geldbörse sich zueignen wollte. Die Warum-Frage des Richters war eine offene Frage, d.h. der Richter hat der Angeklagten keine Antwortmöglichkeiten vorgegeben. Im Rahmen von Shriften zur Interviewtechnik werden nun die Konsequenzen von offenen Fragen diskutiert. Empirische Untersuchungsergebnisse zeigen, daß »bei offenen Fragen relativ häufiger Befragte mit geringer Schulbildung und von

zialforschung, in: René König, Hrsg., Handbuch der empirischen Sozialforschung, Suttgart 1967, Bd. 2, S. 136-97 und S. 707-16. René König, Hrsg., Das Interview, s. Aufl. Köln 1965. 10 Der Leser, der sidh über diese spezielle Soziologie informieren will, sei auf folgende deutschsprachige Beiträge verwiesen: Tilman Moser, Jugendkriminalität und Gesellschaftsstruktur, Frankfure 1970. Karl-Dieter Opp, Kriminalität und Gesellschaftsstruktur. Eine kritische Analyse soziologischer Theorien abweidienden Verhaltens, Neuwied und Berlin I968. Fritz Sack, und René König, Hrsg., Kriminalsoziologie, Frankfurt 1968. Fritz Sadk, Probleme der Kriminalsoziologie, in: René König, Hissg., Handbuch der empirischen Sozialforschung, Bd. z, Stuttgare 1969, S. $961-1049$.

11 Aus der genannten Untersuchung von Opp und Peudsert geht hervor, daß offenbar eine Reihe von Theorien von Richtern relativ einheitlich angewendet werden. 
niedriger sozialer Schiche überfordert werden können «12. D. h. erstens haben Angehörige unterer Schichten Schwierigkeiten, Gefühle usw. zu verbalisieren und zweitens dürften Angehörige der Unterschicht kaum so bewußt ihr eigenes Verhalten beobachten, daß sie über irgendwelche Motive* Auskunft geben können ${ }^{13}$. Die Angeklagte gehörte nun eindeutig der Unterschicht an. Der Tatbestand, daß die Angeklagte die genannte Frage nicht beantworten konnte, wird also ein Soziologe als völlig irrelevant dafür, ob sie die Tat begangen hat oder niche, betrachien.

Eine wichtige Rolle für den Schuldspruch dürfte die im Prozeß unbestrittene Tatsache gespielt haben, daß die Angeklagte dem Bestohlenen die Geldbörse aus der Tasche genommen hatte. Mit einer gegebenen Handlung können nun verschiedene Ziele realisiert werden. Wenn etwa jemand ein Küchenmesser kauf, kann er das Ziel haben, einen deutschen Richter zu ermorden, eine Bitte seiner Frau zu erfüllen oder mit der hübschen Verkäuferin ins Gespräch zu kommen. Der Richter hat nun angenommen, daß die Frau bei der Entnahme der Geldbörse nur ein einziges Ziel hatte, nämlich sich das Geld zuzueignen. Diese These würde ein Soziologe oder Psychologe nur dann akzeptieren, wenn er geprüft hat, ob die genannte Handlung nicht auch für die Realisierung anderer Ziele hätte relevant sein können. Es wäre z. B. möglich gewesen, daß die Angeklagte Prestige bei ihrem Bekannten oder bei anderen Personen, die am Tisch saßen, gewinnen wollte. Der Richter hätte z. B. die Angeklagte über ihr Verhältnis zu diesen Personen erzählen lassen können. Falls das genannte Bedürfnis vorlag, hätte er fragen können, wie die am Tisch sitzenden Personen nach der Meinung der Angeklagten auf das Herausziehen der Geldbörse reagierten, um festzustellen, ob die Angeklagte ihre Handlung als relevant für die Realisierung des Ziels „Prestigegewinn « betrachtete. Wenn der Richter aufgrund der Verbalisierungsschwierigkeiten der Angeklagten Bedürfnisse nicht ermitteln konnte, hätte er sie und Zeugen danach fragen können, welche Handlungen in der Situation, in der sich die Angeklagte befand, üblich sind, anders gesagt: ob die Handlung Teil der positiv bewerteten Aktivitäten im Rahmen der Gruppe war, der die Angeklagte zugehörte. Alle diese Tatbestände hat der Richter nicht festgestellt. Ein Soziologe hätte also die These, daß das Herausnehmen der Geldbörse auf die Absicht, sich die Geldbörse zuzueignen, schließen läßt, nicht akzeptiert, jedenfalls nicht in dieser uneingeschränkten Weise.

Auch die These, die einschlägigen Vorstrafen erhöhren die Wahrscheinlichkeic, daß sich die Angeklagte die Geldbörse zueignen wollte, würde ein Soziologe in dieser Weise nicht akzeptieren. Offenbar wendete der Richter implizit die $\mathrm{Hy}$ pothese an, daß jemand, der zu einem früheren Zeitpunke bei »bestimmter Gelegenheit « das Ziel hatte, sich eine Sache rechtswidrig zuzueignen, dieses Ziel auch zu einem späteren Zeitpunkr bei »bestimmten Gelegenheiten « hat. Daß dies im Falle der Angeklagten zurrifft, hätte eigentlich dem Richter selbst zweifelhaft erscheinen müssen; wir sahen, daß er offenbar die Hypothese akzeptierte, Strafen hätten eine wessernde* Wirkung bei dem Bestraften. Die Angeklagte wurde für die im Strafregister vermerkten Delikte bestraft. Also wäre doch gerade anzunehmen gewesen, daß die Angeklagte nun nicht die Absicht hatte, sich die Geldbörse zuzueignen. Wie dem auch sei: Die Konstanz eines Ziels kann

$12 \mathrm{Vgl.} \mathrm{Erwin} \mathrm{K.} \mathrm{Scheuch,} \mathrm{Das} \mathrm{Interview} \mathrm{in} \mathrm{der} \mathrm{Sozialforshung,} \mathrm{a.a.O.,} \mathrm{S.} 146$.

$13 \mathrm{Vgl}$. hierzu etwa Basil Bernstein, Some Sociological Determinants of Perception. An Inquiry into Sub-Cultural Differences, in: Britisch Journal of Sociology 1958, Bd. 9, S. I 59-74. Siehe insbesondere vom gleichen Autor: Language and Social Class, in: British Journal of Sociology 1960, Bd. $1 x$, S. $271-76$. 
nicht ohne weiteres angenommen werden; es gibt viele Ziele, die sich im Zeitablauf ändern. Ob das Ziel der Angeklagten, sich in bestimmten Situationen rechtswidrig Sachen zuzueignen, konstant geblieben ist oder nicht, hätte man im einzelnen prüfen müssen. Ein Soziologe hätte z. B. zu ermitteln versucht, ob die Angeklagte ihre Bezugsgruppe geändert hat, d. h. ob die Angeklagte an anderen Personen oder Gruppen als vor ihrer letzten Tat ihr Verhalten orientierte.

Befassen wir uns nun mit der Theorie des Richters über die Wirkung der von ihm verhängten Strafe. Zunächst nahm der Richter an, daß das Strafregister die tatsächlich von der Angeklagten begangenen Taten enthält. Dies würde ein Soziologe nicht ohne weiteres akzeptieren. Eine Vielzahl von Untersuchungen besonders in jüngerer Zeit haben ergeben, daß die Dunkelziffer - d. h. die nicht entdeckte Delinquenz - extrem hoch ist, d. h. daß offizielle Unterlagen in dieser Hinsicht völlig unzuverlässig sind ${ }^{14}$. Dabei stellt die entdeckte Delinquenz keineswegs eine Zufallsauswahl der tatsächlichen Delinquenz dar, sondern eine verzerrte Auswahl, d.h. bestimmte Täter haben eine höhere Chance, entdeckt $\mathrm{zu}$ werden, als andere Täter. Es erscheint besonders plausibel, daß die Chance der Angeklagten, während ihrer Ehe entdeckt zu werden, relativ gering war. Der Grund ist, daß die Polizei wahrscheinlich die Heirat als »kriminalitätshemmend" betrachtete und so wohl der Angeklagten weniger Aufmerksamkeit als vor ihrer Ehe widmete. Die Vorstellung, daß eine Heirat kriminalitätsreduzierend wirkt, war auch bei dem Richter vorhanden: Er führte die "Straflosigkeit" auf die Heirat zurück. Wenn diese Vorstellung zutreffen sollte, wäre zu empfehlen, in Zukunft unverheiratete Angeklagte nicht mehr zu Geld- oder Gefängnisstrafen, sondern zum Heiraten zu verurteilen. Es ist also bei der Angeklagten zumindest nicht ausgeschlossen, daß die Straflosigkeit allein im Strafregister stand.

Aber nehmen wir einmal an, die Angeklagte sei wirklich straflos gewesen, so daß für sie gilt: Je höher die Strafe ist, desto geringer ist die Wahrscheinlichkeit, daß die Angeklagte rückfällig wird. Wie würde nun ein Soziologe diese These beurteilen? Zunächst würde man darauf hinweisen, daß allgemein die Rückfälligkeit von Gefängnisinsassen relativ hoch ist. Von da aus ist damit zu rechnen, daß auch die Angeklagte wieder rückfällig wird. Dies hat sich auch bis zu der erneuten Verurteilung bestätigt: Die 30 Strafen haben die Angeklagte offenbar nicht von weiteren Taten abgehalten, wenn man einmal unterstellt, daß zumindest einige Schuldsprüche keine Fehlurteile waren. Es wäre jedoch denkbar, daß die Angeklagte in eine Strafanstalt eingewiesen wird, in der relativ gure Voraussetzungen für späteres straffreies Verhalten geschaffen werden - etwa eine negative Bewertung bestimmter krimineller Aktivitäten. Somit wäre auch die Rückfälligkeitschance der Angeklagten relativ gering einzuschärzen. Der Richter hat sich jedoch nicht darum gekümmert, in welche Art von Gefängnis die Angeklagte eingewiesen werden wird.

Aber selbst wenn die Anstalt, in die die Angeklagte eingewiesen wird, die genannten Voraussetzungen schafft, ist dies nur eine Bedingung für die Chance der Rückfälligkeit nach der Entlassung. Von Bedeutung ist - allgemein gesagt - die Situation, mit der die Angeklagte nach der Entlassung konfrontiert ist. Hier seien nur einige Faktoren angedeutet, deren Vorhandensein ein Soziologe feststellen würde, bevor er eine Aussage über die Rüdkfälligkeitschance der

14 Vgl. hierzu die Literaturangaben in meiner genannten Schrift $>$ Kriminalität und Gesellschaftsstruktur*, Kap. 3. Siehe auch: Stephan und Edelgart Quensel, Delinquenzbelastungsskalen für männliche Jugendliche, in: Kölner Zeirschrift für Soziologie und Sozialpsychologie 1970. Bd. 22, S. $75-97$. 
Angeklagten macht. Es müßten Informationen über die Berufschance der Angeklagten und über die Reaktionen der persönlichen Bekannten vorliegen, etwa inwieweit Kontakte mit ihr vermieden werden bzw. welche Arr von Personen Kontakte mit ihr beenden oder von vornherein nicht aufnehmen, d. h. inwieweit die Strafe eine „Stigmatisierung « verursacht. Dies wäre relevant, um die Personen zu identifizieren, an deren Verhalten sich die Angeklagte wahrscheinlich orientieren wird.

Weiterhin wäre von Bedeutung gewesen, die Vorstellungen bezüglich akzeptablem Verhalten - also die Normen - der genannten Personen zu wissen, um etwa zu erfahren, inwieweit es sich hier um eine »abweichende Subkultur « handeit, in die die Angeklagre nach der Enclassung wieder integrierr wird. Schließlich wäre noch die Ermittlung einiger Einstellungen bzw. Atritüden der Angeklagten von Bedeutung gewesen (einschließlich der möglichen Änderungen im Gefängnis), etwa das Anspruchsniveau bezüglich gewünschrem Einkommen und Arr des gewünschren Berufes oder die Bereitschaft zu einem Ortswechsel. Mit derartigen Tatbeständen hat sich der Richter nichr befaßr. Diese Ausführungen zeigen, daß ein Soziologe auch die These über die Wirksamkeir der Strafe so nicht akzeptiert hätte.

In diesem Prozeß hätte der Soziologe also andere Theorien als der Richter angewender. Dies gilt selbst dann, wenn unsere Explikation der Richtertheorien nicht zutreffen sollte. Der Grund ist, daß der Soziologe aufgrund soziologischer Hypothesen Tarbestände erhoben hätre, an deren Kenntnis der Richter nicht interessiert war, und zu anderen Ergebnissen kam. Somir müssen auch andere Theorien angewender worden sein.

In diesem Zusammenhang sei noch auf einen Tarbestand hingewiesen, der für den beschriebenen und auch für andere Strafprozesse typisch ist. Fast während des gesamten Prozesses befaßte man sich damic, den Sachverhalt zu ermitreln. Wenn nun der Richter das Ziel hat, die künftige Rüdkfälligkeir der Angeklagten zu verhindern - was in unserem Falle im Urreil deurlich zum Ausdruck kam, und auch im StGB geforderr wird -, wäre zu erwarten gewesen, daß er sich nach der Ermittlung des Sachverhaltes intensiv mit der Frage der Rüdkfallwahrscheinlichkeit befaßte. Dies schien den Richter jedoch nicht zu interessieren. Keine einzige Frage an die Angeklagte hatre das künftige Verhalten der Angeklagten zum Gegenstand. Dies ist ein erstaunlicher Tatbestand: Man definiert zwar einerseits die Rüdkfälligkeit von Vorbestraften als ein soziales Problem. Mit der Iösung dieses Problems - d. h. mir Maßnahmen, die Rüdkfälligkeit zu verhindern - befaßr man sich jedoch nicht.

\section{Zur Kritik juristischer Alltagstheorien}

Wenn zwei Gruppen von Theorien sich widersprechen, frage es sich, welche dieser Theorien akzeptiert und welche eliminiert werden sollten. In unserem Falle müßte also entschieden werden, ob man Theorien, die von Juristen im Strafprozeß angewendet werden, oder soziologische Theorien, die diesen juristischen Theorien widersprechen, den Vorzug geben sollte ${ }^{15}$.

Diese Frage wird man u. a. danach entscheiden, welche der Theorien strenger geprüft wurden und sich dabei besser bewährt haben. Inwieweit ist nun dieses

15 Falls widersprühlide soziologische (oder soziologische und sozialpsychologische) Theorien vorliegen, müßre zunächst entschieden werden, welche dieser Theorien akzepciert werden sollen. Die für soldhe Entscheidungen relevanten Kriterien werden im folgenden angedeutet. 
Kriterium bei den juristischen Theorien erfüllt? Welche Untersuchungen können Juristen anführen, in denen die im Strafprozeß angewendeten Theorien geprüft wurden? Welche Fakten stützen z. B. die von unserem Richter angewendete Theorie, daß die Höhe der verhängten Strafe negativ mir der Rückfälligkeitschance korrelierr? In Strafurteilen finden wir üblicherweise keine Untersuchungen angeführt, die die - meist implizit und wohl auch unbewußt - angewendeten Theorien prüfen. Zuweilen wird allgemein auf die "Erfahrung" oder die "Lebenserfahrung « verwiesen. Leider finden wir keine Angaben darüber, auf wessen "Lebenserfahrung" hier Bezug genommen wird. Weiter möchte man gerne wissen, welcher Arr diese Lebenserfahrung ist: Wieviele Personen wurden beobachtet oder befragt? Wie wurden diese Personen ausgewählt? In welcher Weise wurden sie befragt und in welcher Situation wurden sie beobachtet? Solche Fragen werden bei den üblichen Hinweisen auf die "Lebenserfahrung " nich beantworter.

Die Redeweise von der Lebenserfahrung ist also für die Entscheidung, eine Theorie zu akzeptieren oder nicht $\mathrm{zu}$ akzeptieren, so lange irrelevant, als nicht die Art dieser Erfahrung in der beschriebenen Weise spezifiziert wird. Daß die "Lebenserfahrung « von Juristen so häufig ins Feld geführt wird, könnte möglicherweise bezwedken, die angewendeten Theorien vor Kritik zu immunisieren; denn wer wird schließlich die Lebenserfahrung eines ehrenwerten Richters be$z$ weifeln? Aber möglicherweise überschätzt diese Erklärung den Grad, in dem sich die meisten Juristen der Fragwürdigkeit des Geredes von der Lebenserfahrung bewußr sind.

Wir sehen also, daß die Fakten, die den Richter oder andere am Strafprozeß beteiligte Juristen zur Anwendung bestimmter Theorien bewegen, nicht spezifziert sind. Weiterhin ist nicht klar, in welcher Weise die Fakten ermittelt wurden, die die angewendeten Theorien stützen sollen. Niemand weiß also, welche Fakten für die Theorien sprechen und wie diese Fakten erhoben wurden. Der Prozeß der Datenerhebung und der Prozeß der Annahme oder Ablehnung von Theorien ist damit völlig unkontrollierbar ${ }^{10}$.

Diese Strategie öffnet nun Tür und Tor für alle möglichen Fehler. Jeder Soziologiestudent im zweiten Semester weiß, wie stark die Vorgehensweise bei der Datenerhebung - also bei Interview und Beobachtung - das Ergebnis einer Untersuchung beeinflußt. Die Juristen, die ihre Theorien "prüfen«, sind nun üblicherweise nicht in der Erhebung von Daten geschult, so daß die üblichen Fehler, die bei ungeschulten Beobachtern und Interviewern auftreten, auch hier vorkommen dürften. Es hat sich z. B. gezeigt, daß die Kenntnis der zu prüfenden Theorien häufig - bewußt oder unbewußt - dazu führt, daß die Daten in Richtung der Hypothesen verzerrt werden. Der Grund ist, daß der Interviewer, der die zu prüfenden Hypothesen kennt, häufig unbewußt seinen Erwartungen bezüglich solcher Antworten bei bestimmten Interviewfragen Ausdruck gibt, die die Hypothesen bestätigen. Dies hat zur Folge, daß die Antworten der Befragten in der Weise verzerrt werden, daß die zu prüfenden Hypothesen eher bestätigt als widerlegt werden. Man weiß nun nicht nur von Untersuchungen zum Interview, daß Forscher unbewußt ihre Hypothesen zu bestätigen versuchen, also Fakten übersehen, die eine Hypothese widerlegen könnten ${ }^{17}$. Der Jurist

\footnotetext{
16 Auch die Kriminalstatistiken, die zuweilen zur Stützung bestimmter Theorien herangezogen werden, sind keine zuverlässigen Quellen für die Prüfung von Theorien. Dies bat sich vor allem sufgrund der vielen Untersuchungen über die Dunkelziffer gezeigt.

17 Vgl. hierzu etwa viele Hinweise bei Thomas S. Kuhn, Die Strukrur wissenschaftlicher Revolutionen, Frankfurt r 967.
} 
kennt nun die zu prüfenden Hypothesen und "prüft« sie auch selbst. Gerade beim Richter besteht nun eine vorzügliche Gelegenheit, eine Reihe seiner Theorien zu bestätigen. Wenn er z. B. bestimmte Theorien bei der Ermittlung des Sachverhaltes anwendet, dann kann er diese leicht in der Weise bestätigen, daß er Personen schuldig spricht, die bestimmte Merkmale haben; er könnte etwa glauben, daß bei Vorbestraften eine höhere Wahrscheinlichkeit als bei NichtVorbestraften besteht, daß sie im Sinne der Anklage schuldig sind. Kommt nun ein Vorbestrafter vor Gericht, wird er aufgrund der Theorie schuldig gesprochen. Man kann dann z. B. aufgrund der Statistik die genannte Theorie mbestätigen «, da ja tatsächlich Vorbestrafte seltener freigesprochen werden als Nichr-Vorbestrafte.

$\mathrm{Daß}$ die von Juristen verwendete Strategie der "Prüfung ihrer Hypothesen zu unzuverlässigen Ergebnissen führt, wird wohl kaum jemand bestreiten, der die Techniken der empirischen Sozialforschung kennt. Dies kommt u. a. darin zum Ausdruck, daß man üblicherweise irgendwelche vorwissenschaftlichen Vorstellungen eliminiert, wenn sie durch Untersuchungen, die durch die Techniken der empirischen Sozialforschung durchgeführt wurden, widerlegt werden. Wenn also etwa ein Richter glaubt, daß die Höhe der Strafe negativ mit der Rückfälligkeitschance korreliert und wenn diese These durch eine empirische Untersuchung, in der die Techniken der empirischen Sozialforschung dem neuesten Stand gemäß angewendet werden, widerlegt wird, dann wird er seine These eliminieren.

Im Gegensatz zu den beschriebenen juristischen Theorien ist der Prüfungsprozeß soziologischer Theorien weitaus kontrollierbarer. Dies gilt sowohl dann, wenn die Forschungstechnik des Interviews bzw. der schriftlichen Befragung als auch wenn die Technik der Beobachtung angewendet wird. Wir wissen hier zumindest, welche Forscher welche Personen (oder Gruppen) in welcher Weise untersucht haben.

Wir werden also soziologische Theorien, die mittels der Techniken der empirischen Sozialforschung geprüft und sich häufiger bewährt als nicht bewährr haben, konkurrierenden juristischen Alltagstheorien vorziehen.

Dies impliziert selbstverständlich nicht, daß die soziologischen Theorien sich als wahr erwiesen haben oder daß es bezüglich dieser Theorien keinerlei Probleme gibr. Unsere Ausführungen implizieren dagegen nur folgendes: Die soziologischen Theorien mögen noch so schlechr sein: Sie sind in jedem Falle irgendwelchen juristischen Alltagstheorien, die allein aufgrund irgendeines Evidenzgefühls akzeptiert werden, dessen Herkunft man nicht genauer spezifizieren kann und das man vornehm sLebenserfahrung " nennt, überlegen. Bildlich gesprochen: Wenn man ein Auto benötigt und wenn man die Wahl zwischen zwei schlechten Autos hat, wird man nicht auf beide Autos verzichten, sondern man wird das weniger schlechte Auto verwenden.

Es gibt nun eine Vielzahl von soziologischen Theorien, die mittels der Forschungstechniken der empirischen Sozialforschung geprüft und sich relativ gut bewährt haben und auch in Strafprozeß anwendbar sind. Sie werden jedoch wie gesagt - von Juristen im Strafprozeß nicht angewendet. Warum ist dies nicht der Fall? Wenn Juristen glauben, ihre eigenen Theorien seien besser, warum zeigt man dies nicht? Oder warum zeigt man nicht zumindest, daß die soziologischen Theorien völlig unbrauchbar sind? Daß dies nicht geschieht, ist um so erstaunlicher, als man kaum sagen kann, daß die Strafjustiz in der Lage ist, ihre Probleme zu lösen, z. B. daß Verbrechen zumindest bei den verurteilten Tätern verhindert werden. Ein größeres Zeugnis der Unfähigkeit der Justiz als der hier beschriebene Strafrechrsfall ist eigentlich kaum denkbar: Was soll man 
von einer Justiz (im weitesten Sinne dieses Wortes) halten, in der es möglich ist, daß eine Frau $30 \mathrm{mal}$ rüdkfällig wird? Eine soziale Institution ist offenbar nicht in der Lage, die Probleme, deren Lösung von ihr erwartet wird, zu lösen. Sie ignoriert jedoch ein Instrumentarium, das zur Lösung ihrer Probleme beitragen könnte.

Damit diese Ausführungen nicht mißverstanden werden, sei folgendes betont. Wir sind nicht der Ansicht, daß eine Justizreform allein darin bestehen müßte, daß man damit beginnt, sich einmal mit dem relevanten Instrumentarium der empirisch-theoretischen Sozialwissenschaften zu befassen. In welcher Weise man auch immer die Justiz verändern will: In jedem Falle dürften empirische Sätze angewendet werden und wir sind der Meinung, daß diese Sätze explizit formuliert und mit den Mitteln der Sozialwissenschaften überprüft werden müssen, wenn man Fehlentscheidungen vermeiden will. 